ORIGINAL ARTICLE

\title{
Expression of insulin receptor substrate 1 in primary breast cancer and lymph node metastases
}

\author{
M Koda, M Sulkowska, L Kanczuga-Koda, S Sulkowski
}

J Clin Pathol 2005;58:645-649. doi: 10.1136/icp.2004.022590

See end of article for authors' affiliations .....................

Correspondence to: Professor S Sulkowski, Department of Clinical Pathology, Medical University of Bialystok Waszyngtona 13, 15-269 Bialystok, Poland; sulek@ zeus.amb.edu.pl

Accepted for publication 15 November 2004

\begin{abstract}
Background: Insulin receptor substrate 1 (IRS-1) transmits signals from the insulin-like growth factor I receptor (IGF-IR) and insulin receptor (IR) and has been associated with the pathogenesis of cancer. IRS-1 downregulation has been suggested to play a role in breast cancer progression, but no simultaneous assessments of IRS-1 expression in primary breast cancer and metastases have been performed.

Aims: To assess IRS- 1 expression in primary and metastatic breast cancer.

Methods: IRS-1 expression was analysed by means of immunohistochemistry in 109 samples of primary breast cancer and in 42 matched primary and metastatic tumours. In addition, IRS-1 expression was correlated with selected clinicopathological features, including oestrogen receptor $\alpha(E R \alpha)$ and proliferation marker Ki-67 status.

Results: Positive cytoplasmic IRS-1 immunostaining was found in $69.7 \%$ (76 of 109) and $76.2 \%$ (32 of 42) of the primary and metastatic tumours, respectively. Both IRS-1 positive and IRS-1 negative primary tumours produced IRS-1 positive and IRS- 1 negative metastases. IRS- 1 expression in primary tumours correlated with poorly differentiated (G3) breast cancer $(p<0.005)$ and with lymph node involvement $(p<0.05)$. In the subgroup of ER $\alpha$ positive primary tumours, IRS- 1 expression positively correlated with Ki$67(p<0.02, r=0.351)$, but in the subgroup of ER $\alpha$ negative primary tumours there was a negative correlation $(p<0.03, r=-0.509)$. IRS-1 expression in lymph node metastases correlated with neither ER $\alpha$ nor Ki-67.

Conclusions: IRS-1 might be involved in breast cancer progression. Knowledge about differences between primary and metastatic tumours might help to understand mechanisms of breast cancer progression and lead to the development of more effective anticancer drugs.
\end{abstract}

nsulin receptor substrate 1 (IRS-1) is a major signalling molecule of the insulin receptor (IR) and the insulin-like growth factor I (IGF-I) receptor (IGF-IR)..$^{1-3}$ Aberrant expression of IRS-1 has been associated with the pathogenesis of many diseases, including diabetes and cancers of the breast, pancreas, prostate, and liver. ${ }^{2-6}$ Activated IRS-1 transmits signals from IGF-IR and IR by sequestering multiple effector molecules and stimulating different signalling pathways, including the phosphatidyl inositol 3 kinase/ Akt, and extracellular signal regulated $1 / 2$ pathways. ${ }^{137}$ In addition to its conventional role as a cytoplasmic signalling molecule, IRS-1 appears to function in the nuclear compartment to modulate gene transcription..$^{8-10}$

\begin{abstract}
"Aberrant expression of insulin receptor substrate 1 has been associated with the pathogenesis of many diseases, including diabetes and cancers of the breast, pancreas, prostate, and liver"
\end{abstract}

In breast cancer, IRS-1 overexpression has been associated with tumour development, hormone independence, and antioestrogen resistance. ${ }^{6}$ These effects have been attributed to increased tyrosine phosphorylation of IRS-1 and potentiation of its downstream signalling to Akt. ${ }^{6}{ }^{11}$ IRS-1 deficiency in MCF-7 cells was associated with upregulation of oestrogen receptor (ER) protein expression and binding capacity. ${ }^{12}$ In hormone dependent breast cancer cell lines the expression of IRS- 1 has been correlated with $E R \alpha$, and numerous studies have demonstrated that IRS-1 is one of the central elements of ER $\alpha-$ IGF-I crosstalk. ${ }^{611}$ Studies on breast cancer cell lines established that ER $\alpha$ can activate IRS-1 transcription by acting on the IRS-1 promoter. ${ }^{13-15}$ In addition, new data suggest that unliganded ER $\alpha$ can upregulate IGF-I signalling by decreasing IRS-1 degradation through proteasomal pathways. ${ }^{2}$

Despite the apparent importance of IRS-1 signalling in breast cancer, the examination of IRS-1 expression and its correlation with various tumour markers has been described in only a few studies. ${ }^{16}{ }^{17}$ The results of these studies suggested that IRS-1 expression could be involved in the growth regulation of breast cancer, ${ }^{17}$ although Schnarr et al found that IRS-1 downregulation was associated with tumour progression. ${ }^{16}$

The simultaneous assessment of IRS-1 expression in primary breast cancer and metastases has never been performed. In our study, we assessed IRS- 1 expression in primary breast cancer tumours and lymph node metastases and looked for associations between IRS-1 expression and selected clinicopathological features, such as ER status and the proliferation marker Ki-67.

\section{PATIENTS AND METHODS \\ Patients}

Our study comprised 109 women treated surgically with partial or total mastectomy and lymph node dissection for primary breast cancer between 2000 and 2002. Patients received no preoperative chemotherapy or hormonotherapy. Tumour samples were fixed in $10 \%$ buffered formaldehyde solution, embedded in paraffin wax blocks at $56^{\circ} \mathrm{C}$, and routinely stained with haematoxylin and eosin. Histopathological examination was based on the World

Abbreviations: E2, 17 $\beta$-oestradiol; ER $\alpha$, oestrogen receptor $\alpha$; IGF-I, insulin-like growth factor I; IGF-IR, insulin-like growth factor I receptor; IR, insulin receptor; IRS-1, insulin receptor substrate 1 
Health Organisation and pTN classification of breast tumours. ${ }^{18}$ Tumour grade was assessed according to the Bloom and Richardson system. ${ }^{19}$ Our study included only invasive ductal carcinomas: 63 G2 grade and 46 G3 grade. Fifty two of $109(47.7 \%)$ women had involved lymph nodes at the time of diagnosis. The age of the patients ranged from 30 to 82 years (mean, 54.4). The local ethical committee approved the protocol of our study.

\section{Methods}

Immunostaining was performed on two representative sections from the primary tumours and on one to four involved lymph nodes containing the largest metastatic breast carcinoma foci. Immunohistochemical studies were performed using rabbit polyclonal anti-IRS- 1 antibody (Santa Cruz Biotechnology, Santa Cruz, California, USA) at a 1/100 dilution. Expression of ER $\alpha$ and the proliferation marker Ki67 was assessed as described previously. ${ }^{20}{ }^{21}$ Known positive breast cancer specimens were used to determine the optimum primary antibody dilutions. The IRS-1 antibody dilution used resulted in only specific cytoplasmic immunostaining.

The sections were dewaxed in xylene and rehydrated in graded alcohols. Before application of the primary antibody, the sections were heated in a microwave oven at $750 \mathrm{~W}$ for seven minutes in a container with $10 \mathrm{mM}$ sodium citrate buffer, pH 6.0. Sections were allowed to cool in the buffer at room temperature for 30 minutes and were rinsed in deionised $\mathrm{H}_{2} \mathrm{O}$ three times for two minutes each. Endogenous peroxidase was blocked with $1 \%$ hydrogen peroxide for 20 minutes. Non-specific binding was blocked by incubating the slides for one hour with $1.5 \%$ normal blocking serum in phosphate buffered saline. Next, the sections were incubated with the primary antibody at $4^{\circ} \mathrm{C}$ overnight using a staining chamber (The Binding Site, Birmingham, UK). The IRS-1 studies were performed using the avidin-biotin-peroxidase complex (ABC staining system; Santa Cruz Biotechnology) and 3,3'-diaminobenzidine as chromogen. Slides were counterstained with haematoxylin. In the negative controls the primary antibody was omitted.

The expression of IRS-1 was estimated by light microscopy in 10 different tumour fields and the mean percentage of IRS-1 positive tumour cells was scored. The immunoreactivity of IRS-1 was classified as follows: $<10 \%$ positive cells (assessed as negative IRS-1 expression), 10-50\% positive cells, and $>50 \%$ positive cells. In addition, we noticed differences in intensity of immunostaining, so we divided the specimens into groups of weak and strong staining. Scoring sets were as follows: $<10 \%$ positive cells (assessed as negative); $10-50 \%$ positive cells with weak staining; 10$50 \%$ positive cells with strong staining; $>50 \%$ positive cells with weak staining; $>50 \%$ positive cells with strong staining. In view of too few cases in the $>50 \%$ positive cells with weak staining group we joined these cases with the 10$50 \%$ positive cells with strong staining group. Finally, the groups were determined as: $0,<10 \%$ positive cells; $1+, 10-$ $50 \%$ positive cells with weak staining; $2+, 10-50 \%$ positive cells with strong staining or $>50 \%$ positive cells with weak staining; $3+,>50 \%$ positive cells with strong staining. For IRS-1 correlations with clinicopathological features, sections with scores $1+$ or more were taken as positive, whereas those with a score of 0 were taken as negative.

\section{Statistical analysis}

Spearman's test was used to analyse the correlations between IRS-1 expression in primary breast cancer and IRS- 1 in lymph node metastases, between IRS- 1 and $E R \alpha$, and between IRS-1 and Ki-67 expression in primary tumours and lymph node metastases. The correlations of IRS-1 with clinicopathological features were evaluated using the $\chi^{2}$ and Fisher's exact tests. Probabilities of $\mathrm{p}<0.05$ were assumed to be significant.

\section{RESULTS \\ Associations of IRS- 1 with clinicopathological features}

Immunohistochemical analysis of breast cancer sections revealed positive cytoplasmic IRS-1 staining (fig 1) in $69.7 \%$ ( 76 of 109 ) and $76.2 \%$ (32 of 42 ) of the primary and metastatic tumours, respectively. In some tumours, we saw
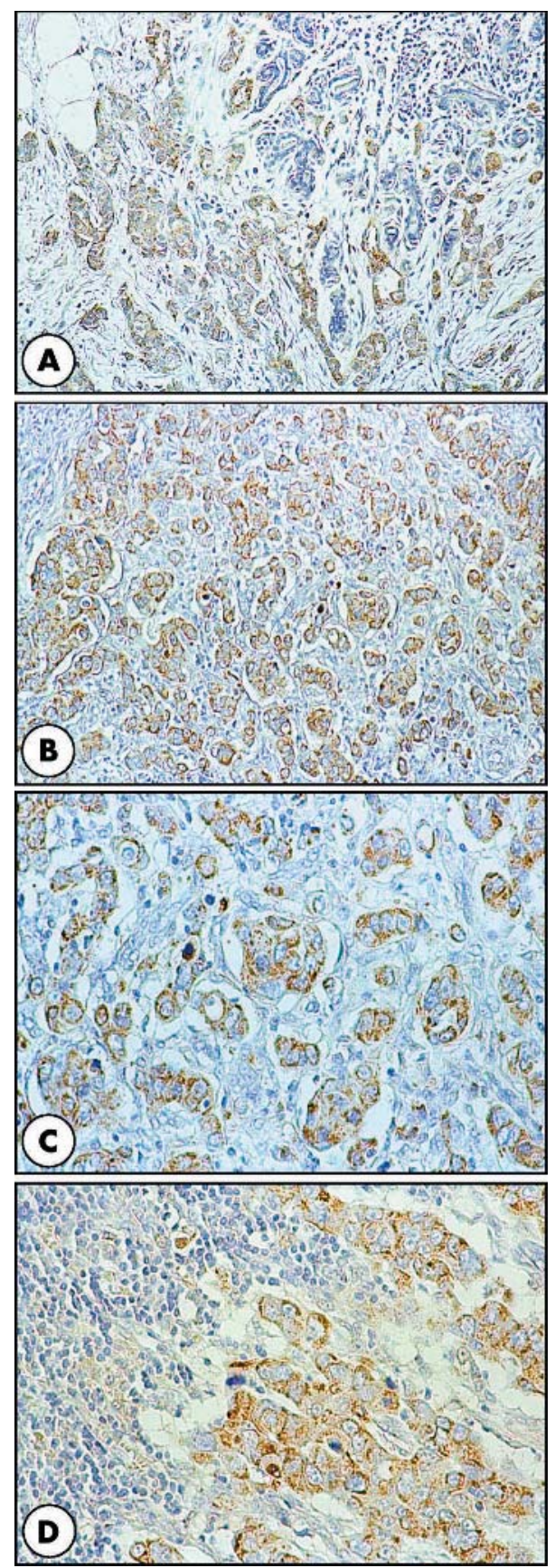

Figure 1 Insulin receptor substrate 1 (IRS-1) expression in breast cancer. (A-C) Positive cytoplasmic and focally perinuclear IRS-1 immunostaining in primary tumour cancer cells. In $(A)$, note the negative IRS-1 immunostaining in the mammary gland adjacent to the cancer. (D) Strong IRS-1 immunostaining in most of the cancer cells in a lymph node metastasis. Original magnification: $A$ and $B, \times 100 ; C$ and $D, \times 200$. 
strong perinuclear localisation of IRS-1. IRS-1 staining was not detected in the negative controls.

IRS-1 expression in breast cancer and in lymph node metastases was associated with poorly differentiated primary tumours (G3) ( $p<0.005$ and $p<0.04$, respectively). IRS- 1 expression in the whole group of primary tumours was not associated with node status $(\mathrm{pN})$, but in the subgroup of better differentiated (G2) cancers, we saw a correlation between IRS-1 expression and lymph node involvement $(p<0.05)$. IRS- 1 expression was not associated with tumour size or patient age.

\section{Expression of IRS- 1 in matched pairs of primary tumours and lymph node metastases}

To assess changes in the expression of IRS-1 during breast cancer progression, it was examined in 42 matched pairs of primary tumours and regional lymph node metastases (table 1 ). In 16 of 42 matched pairs (38.1\%), we found the same degree of IRS-1 staining in both the primary tumours and the lymph node metastases. Increased IRS-1 expression at metastatic sites relative to the primary tumour was found in 14 of 42 cases, and decreased expression in 12 of 42 cases. Five of 30 IRS-1 positive primary tumours had negative metastases and seven of 12 IRS-1 negative primary tumours developed IRS-1 positive metastases.

There was a trend towards a positive association between IRS- 1 expression in primary breast cancer and IRS- 1 expression in lymph node metastases $(\mathrm{p}=0.08, r=0.280)$. In the subgroup of patients with better differentiated tumours (G2), there was a significantly positive correlation $(\mathrm{p}<0.002$, $r=0.664$ ) between IRS-1 expression in the primary and metastatic tumours. This relation was not found in patients with poorly differentiated tumours (G3).

\section{Associations between IRS- 1 and ER $\alpha / \mathrm{Ki}-67$ expression}

ER $\alpha$ and Ki-67 positive cases were found in 66 of $109(60.6 \%)$ and 76 of $109(69.7 \%)$ primary tumours and in 23 of 42 and 28 of 42 metastases, respectively. Comparative studies revealed that in primary tumours and in lymph node metastases, IRS-1 expression did not significantly correlate with ER $\alpha$ or Ki-67 expression. However, in primary tumours we found a trend towards a negative correlation between IRS- 1 and $\operatorname{ER} \alpha(\mathrm{p}=0.061, r=-0.182)$.

Next we assessed the relations between IRS-1 and Ki-67 depending on the ER $\alpha$ status. In ER $\alpha$ positive cases, there was a positive correlation between IRS-1 and Ki-67 $(\mathrm{p}<0.02, r=0.351)$, whereas in ER $\alpha$ negative tumours there was a negative correlation between IRS-1 and Ki-67 $(\mathrm{p}<0.03, r=-0.509)$. We found no associations between IRS-1 and Ki-67 depending on ER $\alpha$ status in lymph node metastases.

\section{DISCUSSION}

Based on cellular models, overexpression of IRS-1 has been implicated in breast cancer development, hormone independence, and antioestrogen resistance, ${ }^{6}$ but only a few reports have shown IRS-1 expression in human clinical material. $^{13} 1617$ Moreover, despite the suggested involvement of IRS-1 in cancer cell motility, ${ }^{5223}$ there are no studies regarding IRS-1 expression in breast cancer metastases. Here, we performed a simultaneous assessment of IRS-1 expression in primary and metastatic breast cancer. To our knowledge, this is the first report of its kind.

Our results indicate a trend $(\mathrm{p}=0.08)$ towards a positive correlation between the expression of IRS-1 in both primary and metastatic breast cancer and a significant $(\mathrm{p}<0.002)$ correlation in the subgroup of patients with G2 grade primary tumours. Moreover, we found an association between IRS-1 expression and poorly differentiated (G3) primary tumours and between IRS-1 expression and lymph node involvement. Despite the similarities in the percentages of IRS-1 positive primary and metastatic tumours, the assessment of IRS- 1 in 42 matched primary and metastatic tumour pairs revealed alterations of IRS-1 expression. Seven of 12 IRS-1 negative primary tumours developed IRS-1 positive metastases, whereas of the 30 IRS-1 positive primary tumours, only five of the matched lymph node metastases were IRS-1 negative. The development of a positive metastasis from a tumour classified as IRS-1 negative is not unusual because most negative tumours contain a small percentage of positive cells that could give rise to a metastatic population. Moreover, it is possible that IRS-1 is upregulated in metastatic sites compared with primary tumours. However, we cannot fully explain the conversion of IRS-1 positive primary tumours into negative metastases. It is possible that part of the primary tumour might show a decrease in IRS-1 expression during breast cancer progression and that the immunohistochemical method used here is not sufficiently sensitive to detect this lower IRS-1 expression.

In vitro studies indicate that IRS-1 is important for cell cycle progression and mitogenic signalling of IGF-I. ${ }^{24}{ }^{25}$ Rocha et al found a lack of correlation between IRS-1 expression and mitotic activity in cancer cells assessed by evaluation of the S-phase fraction, ${ }^{17}$ but they suggested that higher IRS-1 levels enhance cancer growth and make earlier relapse possible. In our study, we found a positive association between IRS- 1 and Ki-67 in ER $\alpha$ positive primary tumours and a negative correlation in ER $\alpha$ negative tumours. Such an association was absent in lymph node metastases. Contrary to Rocha et al, who found an association between IRS-1 and ER expression, ${ }^{17}$ we noted a trend towards a negative correlation between IRS- 1 and ER $\alpha$. Our findings confirmed data obtained in an in vitro study. ${ }^{12}$ However, in the other study on clinical material, IRS-1 expression was downregulated in poorly differentiated tumours. ${ }^{16}$ Moreover,

Table 1 IRS-1 expression in primary tumours and lymph node metastases

\begin{tabular}{llllll}
\hline $\begin{array}{l}\text { IRS-1 expression } \\
\text { in primary } \\
\text { tumours }\end{array}$ & \multicolumn{2}{l}{ IRS-1 in lymph node metastases } & Total number of primary \\
\cline { 2 - 5 } & 0 & $1+$ & $2+$ & $3+$ & tumours \\
\hline 0 & 5 & 1 & 5 & 1 & 12 \\
$1+$ & 2 & 4 & 3 & 1 & 10 \\
$2+$ & 2 & 4 & 6 & 3 & 15 \\
$3+$ & 1 & 0 & 3 & 1 & 5 \\
Total number & 10 & 9 & 17 & 6 & 42 \\
of metastases & & & & & \\
\hline
\end{tabular}

Data are expressed as the number of paired cases in each group. IRS- 1 expression in primary tumours and metastases was assigned on a $0-3+$ scale, as described earlier. IRS-1, insulin receptor substrate 1 . 
Schnarr et al showed a significant increase of Ki-67 labelling in poorly versus well and moderately differentiated tumours, ${ }^{16}$ which was accompanied by decreasing IRS-1 expression, but formal statistical analysis between IRS- 1 and Ki-67 was not performed. In our present study, we found a positive correlation between IRS-1 and Ki-67 in the ER $\alpha$ positive subgroup of primary tumours and a negative association in the $E R \alpha$ negative cancers. We suggest that IRS-1 might produce differential effects on proliferation depending on the ER status, so that a correlation between IRS- 1 and Ki-67 in the whole group of patients cannot be seen. Indeed, differential effects of the IGF-I-IRS-1 pathway have been described in ER $\alpha$ positive and ER $\alpha$ negative breast cancer cells. ${ }^{24}$

Lee et al suggested that tumours expressing both ER and IRS-1 could have a growth advantage, reflected by early recurrence after surgery. ${ }^{13}$ In addition, they reported that the subgroup of ER positive patients with high IRS-1 expression had a significantly shorter disease free survival than those with low IRS-1 expression. In our present study, in the ER $\alpha$ positive subgroup of patients, IRS-1 positively correlated with Ki-67. Notably, Archer et al showed that a high Ki-67 labelling index was associated with a shorter time to progression and overall survival. ${ }^{26}$ This suggests that IRS-1 may be involved in the growth regulation of breast cancer and could predict worse prognosis.

\section{"Our observations suggest that during breast cancer progression increased insulin receptor substrate 1 expres- sion might be implicated in the metastatic process"}

It has been found that $17 \beta$-oestradiol (E2) increased IRS-1 at the mRNA level also, and that antioestrogens reversed the induction. ${ }^{1327} 28$ Similar to cell growth in vitro, a xenograft model of human breast cancer growing in the presence of oestrogen had high levels of IRS-1 expression, in addition to phosphorylated IRS-1 and mitogen activated protein kinase. ${ }^{13}$ In our present study, which included only invasive ductal carcinomas without preoperative chemotherapy or hormonotherapy, we found a trend towards a negative correlation between IRS-1 and ER expression in primary breast cancer. In previous studies, IRS-1 expression positively correlated with ER status, ${ }^{16}{ }^{17}$ but there is a lack of information about preoperative chemotherapy, and the patients were heterogeneous with regard to the histological type of the tumours. In addition, in the study of Schnarr et al a correlation between IRS-1 expression and ER was seen only in grade G2 tumours. ${ }^{16}$ Lee et al found that E2 increased IRS-1 expression, ${ }^{13}$ but the effect of E2 on ER expression was not studied. It was noted that E2 decreased ER expression in breast cancer cells. ${ }^{29}$ Thus, information about increased IRS-1 expression after E2 stimulation and decreased ER expression after E2 stimulation suggests that there may be a negative correlation between IRS-1 and ER. Surmacz and Burgaud reported that in breast cancer cells overexpressing IRS-1,30 oestrogen requirements for growth were reduced and were dependent on the level of overexpression. Our results regarding a negative association between IRS-1 and ER in primary tumours confirm the findings of Ando' et al, ${ }^{12}$ who found that IRS-1 deficiency in breast cancer cells correlated with upregulation of ER expression. Ando' et al suggested that IRS-1 dependent signalling might contribute to the negative regulation of ER expression and function in breast cancer cells. ${ }^{12}$ Moreover, it was suggested that overexpression of IRS-1 in breast cancer could contribute to the development of antioestrogen resistance. ${ }^{27}$ The results of Salerno et al, ${ }^{27}$ our previous studies, and our present study could partly explain the resistance of breast cancer to endocrine treatment. ${ }^{2021}$
Take home messages

- A large proportion of primary and metastatic breast tumours was positive for insulin receptor substrate 1 (IRS-1)

- Both IRS-1 positive and IRS-1 negative primary tumours produced IRS-1 positive and IRS-1 negative metastases

- IRS-1 expression in primary tumours correlated with poorly differentiated (G3) breast cancer and with lymph node involvement

- Thus, IRS-1 may be involved in breast cancer progression and knowledge about differences between primary and metastatic tumours might help to understand mechanisms of breast cancer progression and lead to the development of more effective anticancer drugs

The role of IRS- 1 in breast cancer metastasis is not fully understood. In their in vitro studies, Reiss et al noted that IRS- 1 increased the attachment of metastatic cells in prostate cancer. ${ }^{5}$ Moreover, they suggested that decreased IRS-1 expression in metastatic cell lines might be a mechanism by which cancer cells could metastasise, via decreasing cell adhesion and increasing cell motility. These observations suggest that metastatic breast cancer cells should be IRS-1 negative, but our results clearly show IRS-1 expression in most cases. Our observations suggest that during breast cancer progression increased IRS-1 expression might be implicated in the metastatic process.

Based on in vitro studies, it has recently been reported that IRS- 1 can translocate to the nuclei and nucleoli of cells..$^{8-10} 31$ This process can be enhanced by oncogenes such as simian virus $40 \mathrm{~T}$ antigen, $\mathrm{v}$-src, and activated IGF-IR. ${ }^{9}$ It has been suggested that cytoplasmic and nuclear IRS-1 proteins have different functions. ${ }^{32} \mathrm{Tu}$ et al found that nuclear IRS-1 interacts with nucleolin and the upstream binding factor, which is a nucleolar protein regulating RNA polymerase I activity and ribosomal RNA synthesis. ${ }^{10}$ Morelli et al found that nuclear IRS-1 interacts with $\mathrm{ER} \alpha$ and modulates ER $\alpha$ dependent transcription. ${ }^{31}$ Our immunohistochemical method was adjusted to detect only cytoplasmic IRS-1 protein, but using a different procedure, nuclear IRS-1 in human breast cancer sections can also be observed, as mentioned by Schnarr et al. ${ }^{16}$

We suggest that increased expression of IRS-1 in lymph node metastases may be associated with enhanced turnover of cancer cells and increased risk of distant metastases, so that the prognostic implications of IRS-1 upregulation in lymph node metastases should be evaluated in the future. There may be a need for new alternatives to conventional non-selective treatments, but such therapy should be based on the assessment of primary and metastatic tumours in individual patients to reduce undesirable effects.

\section{ACKNOWLEDGEMENTS}

We are grateful to Dr E Surmacz for critical comments and suggestions and to E Jelska for expert technical assistance.

\section{Authors' affiliations}

M Koda, M Sulkowska, L Kanczuga-Koda, S Sulkowski, Department of Clinical Pathology, Medical University of Bialystok, Waszyngtona 13, 15-269 Bialystok, Poland

\section{REFERENCES}

1 Valentinis B, Baserga R. IGF-I receptor signalling in transformation and differentiation. Mol Pathol 2001;54:133-7. 
2 Morelli C, Garofalo C, Bartucci M, et al. Estrogen receptor- $\alpha$ regulates the degradation of insulin receptor substrates 1 and 2 in breast cancer cells. Oncogene 2003;22:4007-16.

3 Yenush L, White MF. The IRS-signalling system during insulin and cytokine action. Bioessays 1997; 19:491-500.

4 Burks DJ, White MF. IRS proteins and beta-cell function. Diabetes 2001;1:140-5.

5 Reiss K, Wang JY, Romano G, et al. IGF-I receptor signaling in a prostatic cancer cell line with a PTEN mutation. Oncogene 2000;19:2687-94.

6 Surmacz E. Function of the IGF-I receptor in breast cancer. J Mammary Gland Biol Neoplasia 2000;5:95-105

7 Baserga R, Peruzzi F, Reiss K. The IGF-1 receptor in cancer biology. Int J Cancer 2003;107:873-7.

8 Lassak A, Del Valle L, Peruzzi F, et al. Insulin receptor substrate 1 translocation to the nucleus by the human JC virus T-antigen. J Biol Chem 2002;277:17231-8.

9 Prisco M, Santini F, Baffa R, et al. Nuclear translocation of insulin receptor substrate- 1 by the simian virus $40 \mathrm{~T}$ antigen and the activated type 1 insulinlike growth factor receptor. J Biol Chem 2002;277:32078-85.

10 Tu X, Batta P, Innocent N, et al. Nuclear translocation of insulin receptor substrate-1 by oncogenes and IGF-I. Effect on ribosomal RNA synthesis. J Biol Chem 2002;277:44357-65.

11 Sachdev D, Yee D. The IGF system and breast cancer. Endocr Relat Cancer 2001:8:197-209.

12 Ando' S, Panno ML, Salerno M, et al. Role of IRS-1 signaling in insulininduced modulation of estrogen receptors in breast cancer cells. Biochem Biophys Res Commun 1998;253:315-19.

13 Lee AV, Jackson JG, Gooch JL, et al. Enhancement of insulin-like growth factor signaling in human breast cancer: estrogen regulation of insulin receptor substrate-1 expression in vitro and in vivo. Mol Endocrinol 1999;13:787-96.

14 Molloy CA, May FE, Westley BR. Insulin receptor substrate-1 expression is regulated by estrogen in the MCF-7 human breast cancer cell line. J Biol Chem 2000;275: 12565-71.

15 Mauro L, Salerno M, Panno ML, et al. Estradiol increases IRS-1 gene expression and insulin signaling in breast cancer cells. Biochem Biophys Res Commun 2001;288:685-9.

16 Schnarr B, Strunz K, Ohsam J, et al. Down-regulation of insulin-like growth factor-I receptor and insulin receptor substrate-1 expression in advanced human breast cancer. Int J Cancer 2000;89:506-13.

17 Rocha RL, Hilsenbeck SG, Jackson JG, et al. Insulin-like growth factor binding protein-3 and insulin receptor substrate-1 in breast cancer: correlation with clinical parameters and disease-free survival. Clin Cancer Res 1997;3:103-9.
18 Tavassoli FA, Devilee P. Pathology and genetics of tumours of the breast and female genital organs. Lyon: IARC Press, 2003

19 Bloom HJG, Richardson WW. Histological grading and prognosis in breast cancer. Br J Cancer 1957;1 1:359-77.

20 Koda M, Sulkowski S, Garofalo C, et al. Expression of the insulin-like growth factor-I receptor in primary breast cancer and lymph node metastases: correlations with estrogen receptors $\alpha$ and $\beta$. Horm Metab Res 2003;35:794-801.

21 Koda M, Sulkowski S, Kanczuga-Koda L, et al. Expression of ER $\alpha, E R \beta$ and Ki67 in primary tumors and lymph node metastases in breast cancer. Oncol Rep 2004;11:753-9.

22 Reiss K, Wang JY, Romano G, et al. Mechanisms of regulation of cell adhesion and motility by insulin receptor substrate-1 in prostate cancer cells. Oncogene 2001;20:490-500.

23 Nolan MK, Jankowska L, Prisco M, et al. Differential roles of IRS-1 and SHC signaling pathways in breast cancer cells. Int J Cancer 1997;72:828-34.

24 Bartucci M, Morelli C, Mauro L, et al. Differential insulin-like growth factor I receptor signaling and function in estrogen receptor (ER)-positive MCF-7 and ER-negative MDA-MB-231 breast cancer cells. Cancer Res $2001 ; 61: 6747-54$

25 Rose DW, Saltiel AR, Majumdar M, et al. Insulin receptor substrate 1 is required for insulin-mediated mitogenic signal transduction. Proc Natl Acad Sci U S A 1994:91:797-801.

26 Archer SG, Eliopoulos A, Spandidos D, et al. Expression of ras p21, p53 and c-erbB-2 in advanced breast cancer and response to first line hormonal therapy. Br J Cancer 1995; 72:1259-66.

27 Salerno M, Sisci D, Mauro L, et al. Insulin receptor substrate 1 is a target for the pure antiestrogen $\mathrm{ICl} 182,780$ in breast cancer cells. Int $J$ Cancer 1999:81:299-304.

28 Dupont J, Le Roith D. Insulin-like growth factor 1 and oestradiol promote cell proliferation of MCF-7 breast cancer cells: new insights into their synergistic effects. Mol Pathol 2001;54:149-54.

29 Saceda $\mathrm{M}$, Lindsey RK, Solomon $\mathrm{H}$, et al. Estradiol regulates estrogen receptor mRNA stability. J Steroid Biochem Mol Biol 1998;66:113-20.

30 Surmacz E, Burgaud JL. Overexpression of insulin receptor substrate 1 (IRS-1) in the human breast cancer cell line MCF-7 induces loss of estrogen requirements for growth and transformation. Clin Cancer Res 1995; 1:1429-36.

31 Morelli C, Garofalo C, Sisci D, et al. Nuclear insulin receptor substrate 1 interacts with estrogen receptor $\alpha$ at ERE promoters. Oncogene 2004;23:7517-26

32 Tu X, Wu A, Maiorana A, et al. Subcellular localization of IRS-1 in cell proliferation and differentiation. Horm Metab Res 2003;35:734-9. 